\title{
Pharmacist Performance of Physical Assessment: Perspectives of Clinical Pharmacists Working in Different Practice Settings
}

\author{
Doson Chua, Fatima Ladha, Robert T Pammett, and Ricky D Turgeon
}

\section{INTRODUCTION}

$\mathrm{T}$ he scope of practice for pharmacists is rapidly evolving in Canada, with pharmacists in most provinces and territories currently having some degree of prescribing authorization. ${ }^{1}$ In anticipation that this expanded scope of practice would lead pharmacists to seek help in developing more comprehensive assessment skills, the British Columbia Branch of the Canadian Society of Hospital Pharmacists (CSHP) launched the Physical Assessment Course for Pharmacists in September 2015. The course, sponsored in part by the College of Pharmacists of British Columbia, is now offered in 3 cities, with expanded enrolment. (For details about ongoing or upcoming classes, see www.cshp-bc.com/events/events.html.) To date, more than 130 pharmacists have graduated from this course, with many now integrating physical assessment (PA) into their practice.

This article details interviews with 3 clinical pharmacists (D.C., F.L., R.T.P.) who, after completing this course, have integrated PA into their widely different settings. In this article, these pharmacists share information about how they have changed their respective practices by integrating PA, the challenges they have faced, and their advice for pharmacists wishing to do the same.

\section{METHODS}

The fourth author (R.D.T.), who is an instructor for supplemental PA workshops but was not directly involved with the PA course when these interviews were performed, initiated this project. He invited 3 pharmacists to be interviewed to report personal accounts of their recent successful integration of PA into clinical practice following completion of the PA course. This author designed the questions, administered them by e-mail, asked for elaboration of answers when needed, and compiled and edited the manuscript.

\section{INTERVIEW RESPONSES}

Question 1: Describe your practice setting.

Chua: I work in a cardiac intensive care unit (CICU) at a quaternary care academic teaching hospital. I participate in team rounds every morning for approximately 4 hours with the CICU team, composed of a cardiologist, a cardiology fellow, medical residents, medical students, and me. I am also responsible for a 25-bed cardiac step-down unit.

Ladha: I work in an oncology unit and palliative complex care unit at a tertiary care hospital. The oncology unit mainly consists of patients requiring administration of chemotherapy or management of chemotherapy-induced adverse effects. The palliative complex care unit includes patients requiring pain and symptom management related to cancer and other end-stage diseases.

Pammett: I work in multidisciplinary primary care clinics with family physicians, nurses, and allied health care providers. I see patients by appointment, as well as through consultations from other health care professionals. Throughout the day, my nonpharmacist colleagues also rely on me for drug-related queries and for support in decision-making. 
Question 2: When do you perform PA, for which drug do you find it most useful, and which techniques do you routinely perform?

Chua: I routinely join the CICU team in performing PA during rounds. Since I am commonly involved in writing discharge prescriptions, I am often the patient's last point of contact prior to discharge. As such, I use this opportunity to do a final assessment of volume and vital signs to determine home doses of diuretics and other cardiovascular medications.

Furosemide is commonly used in my practice, so I find great value in performing a comprehensive volume assessment composed of monitoring oxygen requirements, identifying jugular venous distension, auscultating for a third heart sound and crackles, and palpating for edema. At times, I perform additional manoeuvres such as lung auscultation to assess for wheezes suggesting asthma or chronic obstructive pulmonary disease (COPD). Because I work in a critical care setting where nurses frequently measure vital signs, I rarely measure them myself. Despite working in a multidisciplinary team, I have found performing PA to be very useful, particularly when there are no physicians available or when I have been delegated the authority to prescribe.

Ladha: I have integrated PA into my patient interviews to enhance history-taking. Due to the subjective nature and waxing-and-waning patterns of patients' symptoms, performing the patient's history and PA myself has improved my ability to deliver individualized pharmaceutical care. I believe we can all recall a time when reliance on another health care provider's assessment, including someone else's examination based on a chart note, resulted in suboptimal therapy decisions. Before learning PA, I often had to wait for someone else to perform PA to complete my patient assessment, which in some cases even resulted in delayed management of oncological emergencies. Notably, I do not perform PA for every patient, every day. I determine which patients will mostly likely benefit from PA as part of their pharmacotherapeutic work-up and monitoring, and balance this with time constraints imposed by my other responsibilities.

I will generally utilize various PA manoeuvres dependent upon the clinical scenario. Opioids, laxatives, antidiarrheal agents, corticosteroids, and anticonvulsants are the most commonly used drugs in my practice, and therefore most of the PA that I perform focuses on monitoring the safety and efficacy of these agents. As it is minimally invasive and can provide critical information about a patient's health status, I find inspection to be the most useful component of PA. I also commonly perform palpation and auscultation, for example, performing lung auscultation to monitor for the resolution of pneumonia. In my practice, I have not found a definitive role for percussion-based manoeuvres, and rarely perform them since I have found them to be distressing to my patient population.
Pammett: I most commonly measure vital signs for conditions such as hypertension, atrial fibrillation, and heart failure. Based on the Hypertension Canada guidelines (http://guidelines.hypertension.ca/diagnosis-assessment/ measuring-blood-pressure/), I generally measure blood pressure using an electronic upper arm device, but can now turn to manual blood pressure measurements when the device malfunctions. Occasionally, patients will also want to compare results obtained with their home device to a manual measurement, which I can perform independently without interrupting the appointment to involve another member of the health care team. I also like to confirm automated measurements of very high values, such as a systolic blood pressure above $180 \mathrm{~mm} \mathrm{Hg}$, with manual measurements before flagging such findings for prescribers, for consideration of drug therapy changes.

\section{Question 3: What has been the most impactful or satisfying intervention that you have made using your PA skills?}

Chua: The best example occurred when I was rounding on patients independently in the cardiac step-down unit while the cardiology team was off-site. A nurse approached me and asked for a salbutamol order for one of our patients with dyspnea. Notably, this patient had been admitted for heart failure with a concomitant history of COPD. My examination of the patient revealed tachypnea with nasal prongs delivering $2 \mathrm{~L}$ of oxygen, down-trending blood pressure, jugular venous distension, loud crackles at the lung bases, and absence of wheeze. Blood pressure was $98 / 69 \mathrm{~mm} \mathrm{Hg}$, which had changed from an admission value of 112/73 $\mathrm{mm} \mathrm{Hg}$.

Based on these findings and an acutely rising serum creatinine concentration, I felt that the patient was experiencing decompensation of heart failure requiring diuretics and inotropes, rather than COPD needing salbutamol. I contacted the cardiology fellow to discuss my findings and asked him to return to re-assess the patient. Later that afternoon, he transferred the patient to CICU, where symptoms were managed with aggressive diuresis and milrinone.

Ladha: My most satisfying PA-based intervention involved a breast cancer patient with brain metastases. Briefly, this patient had been admitted 4 weeks prior to the present admission for witnessed seizure, treated with a phenytoin loading dose followed by $300 \mathrm{mg}$ daily, and was discharged 2 weeks later prior to establishing a therapeutic concentration. Two weeks later, she was readmitted for progressive functional decline.

On admission, the patient's family reported increasing confusion, falls, and distressing visual hallucinations, and the physician noted that she was minimally responsive and ataxic. Goals of care and code status were changed on admission such that bloodwork was to be avoided.

While performing medication reconciliation with the family to identify therapies to be stopped prior to planned 
hospice transfer, the patient opened her eyes to the right to look at a family member, at which point I noticed nystagmus. With permission of the patient's family, I approached her from the right, and confirmed the presence of rapid horizontal nystagmus. I described what I was seeing to the family, and asked if we could order bloodwork to evaluate for phenytoin toxicity based on the patient's lethargy, nystagmus, and ataxia. After the family agreed, I contacted the attending physician, who ordered bloodwork based on my recommendation. The phenytoin concentration was found to be profoundly elevated at $178 \mu \mathrm{mol} / \mathrm{L}$ when corrected for an albumin concentration of $23 \mathrm{~g} / \mathrm{L}$. Based on this finding, the attending physician met with the family to discuss the likelihood that phenytoin toxicity had resulted in the patient's recent functional decline. Within 5 days of holding phenytoin, the patient returned to her prior baseline and was later able to be discharged home rather than sent to hospice care.

Pammett: Ensuring increased blood pressure monitoring has been my most impactful PA-related intervention. My physician and nurse practitioner colleagues previously found it difficult to schedule timely follow-up after changing patients' antihypertensive regimens. Since learning to perform PA, I have helped fill this gap and can offer a follow-up appointment within 2 weeks. At these appointments, I can assess patients for adverse effects and efficacy of the medication, and can also reinforce adherence. If any changes are warranted, I can quickly consult a prescriber to make the appropriate modifications. I am convinced this increased access to monitoring has led to significant improvements in patients reaching individualized blood pressure targets, while minimizing potential adverse drug events.

Question 4: What barriers have you faced and overcome since introducing PA into your practice, and how do your nonpharmacist colleagues respond to you performing PA?

Chua: The greatest barrier that I think we all face when first integrating PA is committing to developing and finetuning PA skills, including asking for help from other health care professionals with more experience in performing PA. To develop my skills, I examine patients with known abnormalities during CICU rounds to identify and recognize the different abnormalities. Additionally, when the cardiology team is doing bedside PA teaching, I ask questions and actively engage in the teaching.

From the beginning, my physician and nursing colleagues were very supportive of pharmacists doing PA. They welcomed it, and even offered to help me improve my PA skills, teach me new PA techniques, and highlight interesting findings. I continue to approach individual cardiologists with my questions, and have even arranged for cardiologists to lead PA teaching for other hospital pharmacists.
Ladha: The main barriers that I faced in the initial stages of integrating PA were concerns by physicians that, as a pharmacist, I was venturing into the role of diagnostician. Some physicians - who had heard that pharmacists were now "pushing for this scope" - went as far as requesting that I not examine patients. I overcame this stigma by continuing to offer PA to my patients that was comprehensive, accurate, and focused on pharmaceutical care. Some of these colleagues eventually turned around, and now welcome me performing PA. This happened progressively over time after proving to them how pharmacist PA can lead to improvements in drug therapy. Even now, others continue to try to dissuade me from performing PA and ignore my findings, including cases where I have been the first to identify red flags for oncological emergencies. Because of the encouraging feedback from other colleagues, I maintain hope that all physicians will eventually value how pharmacist PA can benefit patient care.

Pammett: The biggest barrier that I have faced was my own false assumption that patients would be distrustful of a pharmacist wanting to perform PA. I later recognized that any hesitation and apparent concern from patients was a result of my body language reflecting a lack of confidence in my skills. As I practised performing PA, I became more confident at offering and describing PA to my patients. Now, very few patients even question why a pharmacist would want to examine them.

My physician and nursing colleagues have not had issues with me performing PA. They trust that, since I have chosen to integrate PA into my assessments, I have the competency to do so. In some cases, they duplicate the examination, which I accept as diligent practice rather than lack of confidence in my ability to do PA. I liken it to confirming or repeating certain aspects of a colleague's medication history; some things are worth hearing for yourself.

\section{Question 5: What are ongoing challenges in performing PA in your practice? What do you see as possible solutions?}

Chua: Time is the primary ongoing barrier, largely based on workload demands. I prioritize my PA on the more acutely ill and tenuous patients, in short, patients for whom PA is likely to influence drug therapy decisions.

Ladha: I have experienced resistance from people within our own profession. In my experience, department heads have hesitated to include PA in the job duties for clinical pharmacists, and have yet to formalize support or endorsement for pharmacist PA. This contributes to the perception that PA "is not in the pharmacist's job description".

I believe that the solution to these issues will require a collective effort from all pharmacists. First, those of us who have integrated PA into our practice must persevere in doing so to demonstrate its value. Second, continuing education programs such as the BC Branch Physical Assessment Course 
for Pharmacists must continue to be available to practising pharmacists, and pharmacists must continue to participate in these programs. Third, PA must trickle down into undergraduate, residency, and advanced pharmacy training to truly ingrain this skill into our scope of practice. Finally, this education needs to contextualize PA as part of pharmacotherapeutic decision-making and how it differs from diagnosis.

Pammett: An ongoing challenge for me is correctly interpreting abnormal findings, such as heart murmurs. When uncertain, I ask one of my colleagues to verify or help me interpret the findings, which I can then use as a basis for my pharmaceutical care plan. I enjoy working in a practice setting where I feel comfortable asking another provider to confirm suspicions or provide a second opinion.

\section{Question 6: What is your advice for clinical pharmacists starting to integrate PA into their practice?}

Chua: The best way to start is to jump in! Inform your physician and nursing colleagues that you are learning to perform PA, and ask them if they can teach you or point out interesting findings. The first few weeks will feel a bit awkward and out of your comfort zone, but over time, your confidence will come. If you frame PA in the context of helping with drug therapy decisions, prescribers will understand and appreciate your contributions to performing PA.

Ladha: Talk with your nursing and physician colleagues before you start performing PA. You can get a sense of how they feel about you performing PA and ask if they will support you in developing this skill. Emphasize the unique aspects of pharmacist PA in enhancing monitoring for specific physical manifestations of drug-related adverse effects, and how it can influence and strengthen your drug therapy recommendations. When describing the role of pharmacist PA to nurses and physicians, it may help to explain that you are evaluating patients from a different perspective, not duplicating their exact assessment to identify something that they might have missed or performing a diagnosis.

Pammett: A good first step in integrating PA into your practice is obtaining buy-in from other providers. It also helps to have 1 or 2 other people you can look to as mentors and ask for confirmatory assessment. Ask other providers to bring you into the room (with patient consent) when they discover something interesting. Like all other assessment skills, PA techniques are refined over time with practice and experience.

\section{IMPLICATIONS}

In the course of this project, we identified multiple similarities in the perspectives of 3 pharmacists who have recently integrated PA into their clinical practice. First, although all 3 pharmacists initially faced at least one of the barriers previously identified in a survey of other pharmacists, ${ }^{2}$ these barriers did not prevent successful integration of PA. Second, all 3 pharmacists described and illustrated how the conscientious use of PA resulted in more effective and efficient care. Finally, all 3 pharmacists highlighted the importance of engaging other members of their health care team to define the role of pharmacist-performed PA, dispel misconceptions, and foster collaboration. We believe that these perspectives and stories illustrate what is possible with pharmacist-performed PA, and should serve as a catalyst for more pharmacists to embrace PA as part of their scope of practice.

\section{References}

1. Pharmacists' expanded scope of practice. Ottawa (ON): Canadian Pharmacists Association; 2016 [cited 2016 Nov 24]. Available from: https://www. pharmacists.ca/pharmacy-in-canada/scope-of-practice-canada/

2. Barry AR, McCarthy L, Nelson CL, Pearson GJ. An evaluation of teaching physical examination to pharmacists. Can Pharm J. 2012;145(4):174-9.e1.

Doson Chua, BSC(Pharm), PharmD, BCPS (AQ Cardiology), is a Clinical Pharmacy Specialist, Cardiology, with St Paul's Hospital, and a Clinical Professor with the Faculty of Pharmaceutical Sciences, The University of British Columbia, Vancouver, British Columbia.

Fatima Ladha, BSc, BSC(Pharm), is a Clinical Pharmacist in Palliative Complex Care and Oncology, Surrey Memorial Hospital, Surrey, British Columbia, and a Clinical Instructor with the Faculty of Pharmaceutical Sciences, The University of British Columbia, Vancouver, British Columbia.

Robert T Pammett, BSC, BSP, MSc, is a Research and Development Pharmacist - Primary Care, Northern Health, Prince George, British Columbia, and an Assistant Professor (Partner) with the Faculty of Pharmaceutical Sciences, The University of British Columbia, Vancouver, British Columbia.

Ricky D Turgeon, BSc(Pharm), ACPR, PharmD, is a Post-Doctoral Fellow with the Department of Medicine (Division of Cardiology), University of Alberta, Edmonton, Alberta.

Competing interests: Ricky Turgeon is an instructor for supplemental physical assessment (PA) workshops; however, he was not directly involved with the PA course offered by the BC Branch of the Canadian Society of Hospital Pharmacists at the time when the interviews reported here were performed. No other competing interests were declared.

\section{Address correspondence to:}

Dr Ricky D Turgeon

Department of Medicine (Division of Cardiology)

University of Alberta

2C2 WMC

8440-112 Street NW

Edmonton $A B$ T6G 2B7

e-mail: rturgeon@ualberta.ca

Funding: None received. 